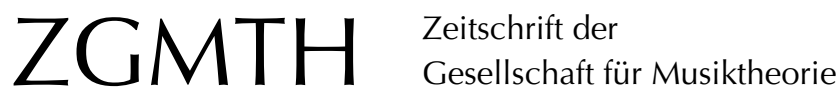

Noble, Andrew R. (2011): Vlitakis, Emmanouil, Funktion und Farbe. Klang und Instrumentation in ausgewählten Kompositionen der zweiten Hälfte des 20. Jahrhunderts: Lachenmann - Boulez - Ligeti - Grisey (= sinefonia 11), Wolke Verlag, Hofheim 2008. ZGMTH 8/2, 381-384. https://doi.org/10.31751/637

(C) 2011 Andrew R. Noble

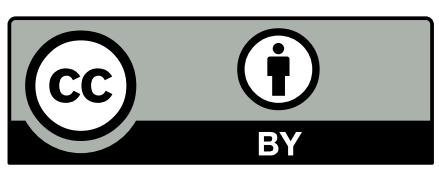

Dieser Text erscheint im Open Access und ist lizenziert unter einer Creative Commons Namensnennung 4.0 International Lizenz.

This is an open access article licensed under a

Creative Commons Attribution 4.0 International License.

veröffentlicht / first published: 17/11/2011

zuletzt geändert / last updated: 25/10/2013 


\section{Emmanouil Vlitakis, Funktion und Farbe. Klang und Instrumentation in ausgewählten Kompositionen der zweiten Hälfte des 20. Jahrhunderts: Lachenmann - Boulez - Ligeti - Grisey (= sinefonia 11), Wolke Verlag, Hofheim 2008}

Wie bereits der Titel verdeutlicht, untersucht Emmanouil Vlitakis in seiner Dissertation »Entwicklungen im Klangdenken nach dem Zweiten Weltkrieg« (11). Sein Interesse gilt vor allem kompositorischen Verfahren, die Klang und Klangfarbe als funktionelle Elemente behandeln und damit neue Formen des musikalischen Zusammenhangs ermöglichen. Es ist das Ziel der Arbeit, anhand ausgesuchter Werke, die nach Meinung des Autors eine "Schlüsselposition innerhalb dieser Entwicklung einnehmen« (13), »die Prinzipien ihrer (vor allem klanglichen) Organisation zu entschlüsseln und möglicherweise das jeweilige Klangdenken und die damit verbundenen kompositorischen Absichten zu rekonstruieren« (13). Im Hinblick auf zukünftige Kompositionen möchte der Autor darüber hinaus „Elemente zur Diskussion zu stellen, welche [...] eine Entwicklungsperspektive haben«. Es gelte, das »bisher Komponierte unter dem Aspekt von Klang und Instrumentation differenzierter wahrzunehmen und seine Entwicklungsmöglichkeiten zu erkennen« (21).

Im Gegensatz zu zahllosen Lehr- und Handbüchern, die das Thema Klang und Klangfarbe in der zweiten Hälfte des 20. Jahrhunderts als Anleitung zur Orchestration behandeln, hat Vlitakis eine wissenschaftliche Untersuchung vorgelegt, in der Klangfarbe als formbestimmender Parameter betrachtet wird. Untersucht wird eine Reihe von Orchesterwerken, zu der mit Griseys Quatre chants pour franchir le seuil ein kammermusikalisches Werk mit vokalen Anteilen hinzutritt. Die behandelten Gestaltungsmerkmale sind Klangfarbe, Tonraum (Tonlage), Dynamik und Artikulation. Dabei zielt die Untersuchung auf folgende Aspekte:
1. »das Moment der Auseinandersetzung zwischen Tonsatz oder kompositorischen Vorformungen des Materials und klanglicher Realisierung",

2. »Momente, in denen das kompositorische Denken primär vom Klang bzw. von der Schallquelle ausgeht",

3. Instrumentationstechniken, "Strategien des Umgangs mit dem Orchester", und

4. den Zusammenhang von Klangfarbe und Text (nur Grisey) (15f.).

Der Hauptteil des Buches besteht aus vier in sich geschlossenen Kapiteln, die durch einen "Prolog" und einen "Epilog" gerahmt sind. Die Kapitel sind jeweils einer Komposition bzw. einer Reihe von Kompositionen eines bestimmten Komponisten gewidmet: Den Anfang macht Lachenmanns Tableau, gefolgt von Boulez' Bearbeitung seiner Notations. Unter der Überschrift »Aspekte des Orchesterwerks" wendet sich Vlitakis Ligetis Apparitions, San Francisco Polyphony, Atmosphères, Lontano, Klavierkonzert und Violinkonzert zu. Den Abschluss bildet Griseys Quatre chants pour franchir le seuil (I).

Am Beginn des Lachenmann-Kapitels bietet Vlitakis eine spannende formale Gliederung des Orchesterstücks Tableau, die »von einer Phänomenologie der Klanggestik ausgeht und die globalen Zusammenhänge der Form aufgrund dieser Phänomenologie andeutet» (26). Die formale Gliederung erfolgt aufgrund des Verhältnisses zwischen »überwiegende[r] Tonlichkeit« (im ersten und dritten Teil) und »überwiegende[r] Tonlosigkeit» (im zweiten Teil). Auf diese Gliederung kommen die folgenden Unterkapitel immer wieder zurück. Besonders überzeugend ist die Diskussion 
der formalen Übergänge. Hier wird gezeigt, wie Verbindungen, Brüche, Verwandtschaften und Kontraste nicht auf der Ebene von Harmonik oder Motivik, sondern der klanglichen Objektbildung realisiert werden.

Unter der Überschrift »Kriterien der Analyse« führt Vlitakis die Bezugspunkte seiner Untersuchung zu Harmonik (»ZweiklangIntervallik«, "tradierte Akkordik", "atonale Klangaggregate«, »Clusterbildungen«, welche vor allem als Mixturbildungen aus Addition tradierter Akkordbildungen entstehen) einschließlich der »Positionierung der Klänge im Tonraum« (sowohl im gesamten Tonraum als auch in dem des jeweiligen Instruments), Dynamik und Zeitgestalt (»Dauer der Einzelelemente«, „Ereignisdichte«, „zeitliches Verhältnis der Elemente und der Abschnitte zueinander") und Klangfarbe (»Teilung in Instrumentengruppen", »registerabhängige Charakteristik«, „Helligkeit/Dunkelheit, Schärfe/Weichheit«, »Ortung«) auf. Als Typen der Objektbildung unterscheidet Vlitakis "grundsätzlich zwischen Fläche und Impuls". Unter »Fläche« versteht er »ausgehaltene Klänge«, »punktuelle Klänge« sowie »Klänge, bei denen einem einzelnen Anschlag ein unterschiedlich langer Nachklang folgt«, unter "Impuls« wird zwischen zahlreichen unterschiedlichen "Klanghervorbringungen" von »minimaler zeitlicher Ausdehnung" differenziert. Allein hinsichtlich der Saiteninstrumente unterscheidet Vlitakis »angeschlagene«, »angehämmerte«, "gestrichene«, "gezupfte« und "getriebene Saiten« (29-42).

Dass Vlitakis' Typologie eine hohe Relevanz für die kompositorische Gestaltung in der zweiten Hälfte des 20. Jahrhunderts beanspruchen darf, zeigt die Diskussion der anderen Kompositionen.

Ein zentraler Aspekt im Schaffen von Pierre Boulez ist das Weiter- und Neu-Komponieren eigener Werke. In dieser Hinsicht erscheint im Kapitel über Boulez das Orchesterwerk Notations I-IV, das aus dem früheren Klavierwerk Douze Notations hervorgegangen ist, gut gewählt (die später bearbeitete VII. Notation wird in der Arbeit nicht behandelt). In kurzen Analysen stellt Vlitakis zunächst einige Haupt- elemente der Klavier-Textur (Ostinato, Glissando, Cluster, usw.) sowie die der Komposition zugrunde liegende Zwölftonreihe dar. Im Anschluss zeigt er, welche Texturelemente als Ansatzpunkte für die kompositorischen Erweiterungen im Orchestersatz dienen und wie sie auf das Orchester übertragen werden (die Orchestersätze sind nicht nur länger, sondern auch komplexer als die Klavierminiaturen).

Ein einfaches Beispiel bietet Notation II, in der die Klavier-Glissandi unter Verwendung des ganzen Orchesterapparats durch eine "glissandierende Klangmasse " aus mehrfachen Linien in unterschiedlichen Stimmen und mit rhythmischen Reibungen (Achtel, Triolen, Sechszehnteln) in schnellem Tempo ersetzt werden, wodurch »der horizontalen [...] eine vertikale Dimension hinzugefügt wird « (81). Als eine interessante Spielart der Erweiterungstechnik erweist sich auch die Vervielfachung des Ostinatos im Klaviersatz zu fünf Schichten im Orchestersatz, die sich durch Klangfarben-Kombinatorik und »nicht durch strikt unterschiedliche Klanglichkeit kontrapunktisch voneinander abheben « (86). Vlitakis legt dar, wie das einfache Ostinato durch die musikalische Erweiterung ausdehnt und so an das neue Medium des Orchesters angepasst wird. Eine Diskussion der Ostinato-Behandlung in Notation $I V$, die sich wesentlich von derjenigen der Notation II unterscheidet, veranschaulicht nochmals die vielfältigen Möglichkeiten des Erweiterns und deren gemeinsame Wurzel im orchestralen (d.h. im medium-spezifischen) Denken. Dabei liegt das Augenmerk der Analyse zunächst auf dem Tonmaterial der Klaviervorlage, das durch Artikulation, Dynamik, Instrumentation usw. markiert wird, um dann die Art der Beziehungen zum Tonmaterial in den orchestralen Erweiterungen aufzudecken. Zahlreiche Tabellen machen die Analyseergebnisse übersichtlich.

Das umfangreichste Kapitel des Buches ist Ligeti gewidmet (131-204). Anhand des ersten Satzes von Apparitions werden zunächst jene Klangflächen behandelt, die für Ligetis Musik vom Ende der 1950er bis in die 1970er Jahre hinein prägend gewesen sind. Die Ka- 
tegorien der Analyse derartiger Klangflächen (»Positionierung im musikalischen Raum«, "Breite« und »Dichte«, »innere Beschaffenheit", "neutraler oder intervallbezogener Aufbau«, "Dynamik«, "Geräuschhaftigkeit», »Bewegungsart oder Statik«) werden anhand konkreter Beispiele dargelegt. Der Charakter dieses Abschnitts ist aber eher zusammenfassend, da die genannten Kategorien bereits aus der einschlägigen Literatur zu Ligetis Musik bekannt sind.

In Anlehnung an Christian Martin Schmidts Aufsatz "Zum Aspekt des musikalischen Raums bei Ligeti « ${ }^{1}$ folgt eine Diskussion der räumlichen Aspekte in San Francisco Polyphony. Von besonderem Interesse ist hier die Andeutung einer "Verbindung und Wechselbeziehung" zwischen »Tiefendimension und Tonhöhenorganisation" (152). Als Beispiel dient der Anfang der Komposition, wo nach einer Phase der Statik eine plötzliche Bewegung und ein Accelerando des harmonischen Rhythmus' den vorwärts drängenden Impuls der Takte 7-10 hervorrufen.

Wer glaubt, die Forschung zu Ligetis monumentalen Klangfarbenkompositionen Atmosphères und Lontano habe sich bereits erschöpft, wird durch Vlitakis' Untersuchungen zum Prinzip der Oktavierung eines Besseren belehrt. Durch die Auffassung der Oktave als Registrierung - das Orchester wird metaphorisch als »virtuelle Orgel « (164-168) beschrieben - werden in Atmosphères Phänomene wie das zeitweise dynamische Hervortreten diatonischer Teilmomente in einer überwiegend chromatischen Fläche erklärt. Der Vergleich zwischen Orchester und Orgel trifft sich mit Zsigmond Szathmárys Bemerkung, dass Ligeti »nur die Prinzipien seiner statischen Musik, wie er sie schon in Atmosphères angewandt hatte, auf Volumina übertragen musste. Diese Prinzipien und diese neue Technik passten ${\text { zueinander } .^{2}}^{2}$

1 Schmidt 1987.

2 Szathmáry 1987, 216. Vgl. auch Ligeti in seiner Analyse des ersten Satzes von Kurtags ... quasi una Fantasia ... (2007).
Auf die Kategorisierung der Klangflächen folgt diejenige der Mixturklänge, wie sie sich im Klavierkonzert und im Violinkonzert finden. Hierbei dürfte es sich um die erste Kategorisierung dieser Phänomene handeln. Zwar ergeben sich hierdurch wertvolle Einblicke in Ligetis funktionelle Verwendung von Mixturklängen, doch bleibt die Chance ungenutzt, eine einheitliche Entwicklungslinie in Ligetis Klangdenken aufzuzeigen. Dies ist darauf zurückzuführen, dass Vlitakis die Mixturenbildung und die Oktavierung als unterschiedliche Prinzipien auffasst statt als unterschiedliche Manifestationen eines einzigen Prinzips.

In vielerlei Hinsicht fällt das erste der Quatre Chants pour franchir le seuil (»D'après Les heures à la nuit de Guez Ricord«), das im letzten Kapitel untersucht wird, aus der Reihe der betrachteten Kompositionen heraus. Dieses letzte vollendete Werk Griseys wurde nicht für Orchester, sondern für Stimme und 15 Instrumentalisten geschrieben. Zunächst diskutiert Vlitakis die Zusammensetzung des Ensembles, die Verteilung und die Hierarchie der vier Instrumentalgruppen (»Hauptgruppe« mit Singstimme und drei »Nebengruppen«). Danach erläutert der Autor das Verhältnis der Instrumentierung zum Text. Er zeigt, wie ursprünglich fixierte Koppelungen zwischen den Klängen der »Hauptgruppe« und bestimmten Textpassagen sich im Dienste einer narrativen Struktur nach und nach ändern.

Eine Behauptung wie »Die Stimme/Trompete-Kombination steht hier eindeutig für $\mathrm{Hel}$ ligkeit und Brillanz [...], Fülle und Bewegung, die Stimme/Altflöte-Kombination dagegen für Statik, Dunkelheit, für einen quasi hauchigen Schattenklang« (221) mag zunächst befremden, bestätigt sich jedoch am Ende der Analyse auf überraschende Weise (223):

"Die Engelhaftigkeit dieses Todes wird im letzten Abschnitt des Stücks besungen, wobei eine völlig neue instrumentale Konstellation erscheint: Die Stimme wird mit den Gruppen 1-3 zusammengeführt, sie wird in die >Begleitungsgruppen integriert, wobei auch die rhythmischen Reibungen zwischen den Melodieinstrumenten geglättet werden, denn 
es entsteht eine einheitliche Achtelbewegung. Gleichzeitig werden die Instrumente der Stimmen-Gruppe ausgeschaltet, die Stimme erklingt jetzt allein, und zwar in einem anderen 'Raum ‘ geortet. Wenn die Stimmengruppe (durch Klangfarbe, Instrumentenumfang und Positionierung im Raum) als klanglicher und symbolischer , Vordergrund gedeutet werden könnte, wobei die Gruppen 1-3 entsprechend einen Hintergrund bildeten, dann findet am Ende des Stücks eine räumliche Verlagerung statt: Die Stimme hat einen neuen Ort erreicht, sie ist nicht mehr da, wo sie war, sondern hat die svordere Welt verlassen und ist singend zu einem Teil der shinteren Welt، geworden."

Wer sich für das Schaffen der behandelten Komponisten interessiert, findet in Vlitakis' Publikation eine Fülle erhellender Analyseansätze, die für eine weitergehende Beschäftigung fruchtbar sein dürften. Seine Analysemethoden erweisen sich als breit genug gefasst, um in den unterschiedlichsten Kontexten verwendet werden zu können, und mit Sicherheit könnte auch die Analyse weiterer gemeinhin als Klangfarbenkompositionen bezeichneter Werke von ihnen profitieren, beispielsweise Pendereckis Threnos oder Cerhas Mouvements. Auch eine gewinnbringende kompo- sitorische Rezeption ist vorstellbar. Insofern ist der vom Autor formulierte Anspruch, eine Entwicklungsperspektive zu geben, eingelöst.

Freilich, wer nicht an die von Vlitakis behauptete Entwicklung glaubt (zumindest nicht in dieser Form), dem wird manche Bemerkung problematisch erscheinen. Dass "Ligeti in San Francisco Polyphony die Tiefendimension des musikalischen Raums wieder aktiviert", nachdem die serielle Musik sie »eher neutralisiert als weiterentwickelt» habe (156), oder dass »nach der 'Läuterung der Klangfarbe von alten Inhalten und der Entwicklung ihres immanenten Potentials" Grisey in den Quatre Chants »eine Synthese auf höherer Ebene" gelingt, in der vorher Verpöntes wieder möglich geworden ist - „Die Klangfarbe darf wieder semantisch eingesetzt werden « (233) -, sind Behauptungen, die Vlitakis kaum zu erweisen vermag, sondern aus einem impliziten Verlaufsmodell folgert, das die Einzelwerke durch einen bestimmten Entwicklungsgang zu verbinden sucht. Aber so problematisch die hieraus resultierenden Einschätzungen hier und da auch sein mögen: Jede der Analysen des Buches ist für sich interessant und lesenswert. Vlitakis' Arbeit verdient Beachtung.

Andrew R. Noble

\section{Literatur}

Ligeti, Gyorgy (2007), Gesammelte Schriften Bd. I, hg. von Monika Lichtenfeld, Mainz u. a.: Schott, 490-496.

Schmidt, Christian Martin (1987), "Zum Aspekt des musikalischen Raums bei Ligeti«, in: György Ligeti: Personalstil-Avantgardismus-Popularität, hg. von Otto Kolleritsch (= Studien zur Wertungsforschung 19), Wien u. a.: Universal Edition, 60-67.
Szathmáry, Zsigmond (1987), „Die Orgelwerke von György Ligeti«, in: György Ligeti: Personalstil-Avantgardismus-Popularität, hg. von Otto Kolleritsch (= Studien zur Wertungsforschung 19), Wien u.a.: Universal Edition, 213-220. 